\title{
Trigeminal Ganglion/Trigeminal Nerve
}

National Cancer Institute

\section{Source}

National Cancer Institute. Trigeminal Ganglion/Trigeminal Nerve. NCI Thesaurus. Code C92214.

A tissue sample that contains the trigeminal gang lion and branches of the trigeminal nerve. 